\title{
Using Ultrasonography to Determine Optimal Head-down Tilt Position Angle in Patients before Catheterization of the Internal Jugular Vein
}

\author{
Anton A. Kasatkin ${ }^{1,2}$, Aleksandr L. Urakov', Anna R. Nigmatullina² \\ 'Department of General and Clinical Pharmacology, Izhevsk State Medical Academy, ${ }^{2}$ Department of Anesthesiology and Intensive Care, Clinical Hospital, \\ Izhevsk, Russia
}

\section{Abstract}

Context: It is believed that $15^{\circ}-25^{\circ}$ head-down tilt position increases the internal jugular vein cross-sectional area (IJV CSA). The increase in IJV CSA before puncture reduces the risk of its perforation. This pattern was not observed in all patients. We assumed that the absence of respiratory-based IJV excursion is one of the criteria of head-down tilt position effectiveness. Aims: The aim of this study is to determine the head-down tilt angle, which ensures the absence of the respiratory-based IJV excursion. Subjects and Methods: Prospective study included twenty adult patients. The IJVs scanning was carried out in 1 min after placing the patients in a horizontal position on their back and in 1 min after placing them in the head-down tilt position at $5^{\circ}, 10^{\circ}, 15^{\circ}$, and $20^{\circ}$ tilt angles. Results: We found that collapsibility index of $<9 \%$ indicating the absence of respiratory-based IJV excursion was recorded in $25 \%$ of patients in the horizontal supine position. In this case, placing the patients in the Trendelenburg position for IJV catheterization may not be indicated. In $65 \%$ of the patients, the respiratory-based excursion was not observed at $10^{\circ}$ head-down tilt position. Only $35 \%$ of the patients required $15^{\circ}$ head-down tilt position. Conclusions: In clinical settings, the disappearance of respiratory-based vein excursion on the ultrasound scanner screen can be considered as criteria of the head-down tilt position effectiveness.

Keywords: Internal jugular vein, respiratory-based internal jugular vein excursion, ultrasound scanning

\section{INTRODUCTION}

To facilitate safe and effective central venous catheterization, in particular, the catheterization of internal jugular vein (IJV), patients are often placed in the head-down tilt position. ${ }^{[1]}$ It is believed that $15^{\circ}-25^{\circ}$ head-down tilt position increases the IJV cross-sectional area (CSA). ${ }^{[2]}$ The increase in IJV CSA before puncture reduces the risk of its perforation and damage to arteries, nerves and pleura, and prevents venous air embolism. It is found that the increase in IJV CSA in the head-down tilt position is observed in patients with a body mass index (BMI) of $<30 \mathrm{~kg} / \mathrm{m}^{2}$. This pattern was not observed in obese patients. In this regard, using the absolute value of IJV diameter to evaluate the necessity of placing a patient in the head-down tilt position and determine its efficiency is acceptable not for all patients. ${ }^{[3]}$ We assumed that the absence of respiratory-based IJV excursion is one of the criteria of head-down tilt position

\begin{tabular}{|l|l|}
\hline \multicolumn{3}{|c|}{ Access this article online } \\
\hline Quick Response Code: & Website: \\
& www.ijccm.org \\
& \\
\end{tabular}

effectiveness. Respiratory-based IJV excursion is a change in its diameter based on the respiration phase. ${ }^{[4]}$ Thus, vein diameter decreases during inhalation and increases during exhalation. It has been shown previously that respiratory-based IJV excursion may impede its safe and effective cannulation. ${ }^{[5]}$ However, currently, there are no scientific data that demonstrate how the head-down tilt position affects respiratory-based venous excursion.

The aim of this study is to determine the head-down tilt angle, which ensures the absence of the respiratory-based IJV excursion.

Address for correspondence: Dr. Anton A. Kasatkin Karl Marks Street 278-45, 426008 Izhevsk, Russia. E-mail: ant-kasatkin@yandex.ru

This is an open access article distributed under the terms of the Creative Commons Attribution-NonCommercial-ShareAlike 3.0 License, which allows others to remix, tweak, and build upon the work non-commercially, as long as the author is credited and the new creations are licensed under the identical terms.

For reprints contact: reprints@medknow.com

How to cite this article: Kasatkin AA, Urakov AL, Nigmatullina AR. Using ultrasonography to determine optimal head-down tilt position angle in patients before catheterization of the internal jugular vein. Indian J Crit Care Med 2017;21:160-2. 


\section{Subjects and Methods}

Approval for this study was obtained from the Ethics Committee based on the principles set in the World Medical Association Declaration of Helsinki. This prospective study included twenty adult patients. Written informed consent was obtained from all participants. Exclusion criteria were BMI more than $30 \mathrm{~kg} / \mathrm{m}^{2}$, inability of the patient to tolerate supine positioning, lack of patient cooperation, pregnancy, severe aortic stenosis, and intracranial and intraocular pressure. All patients involved in the study were in need of central venous catheterization; they were able to make contact (level of consciousness was assessed as 14-15 on the Glasgow coma scale) and did not require lung ventilation. The patients did not receive sedation. Ultrasound scanning of IJV was performed using Siemens Acuson S2000 Ultrasound System (USA) and 5-14 MHz linear transducer. The study was conducted on a hospital bed Futura Plus by Merivaara Company (Finland). The IJVs scanning was carried out in $1 \mathrm{~min}$ after placing the patients in a horizontal supine position and in $1 \mathrm{~min}$ after placing them in the head-down tilt position at $5^{\circ}, 10^{\circ}, 15^{\circ}$, and $20^{\circ}$ tilt angles. Stabilization of hemodynamic was achieved by keeping a patient in a selected position for at least $1 \mathrm{~min}^{\left[{ }^{[6]}\right.}$ The ultrasonic transducer was mounted on the patient's neck between the heads of sternocleidomastoid muscle, and veins cross-scanning was carried out on both sides. The vein diameter sizes during inhalation (maximum) and exhalation (minimum) were recorded through M-mode assessment. All measurements were made by the same physician. The respiratory-based venous excursion was recorded when the difference between the maximum and minimum values of the vein diameter was more than $10 \%$. Quantitative data are presented as mean \pm standard deviation, median, range, and collapsibility index. Statistical processing of data was performed using STATISTICA 10 software (StatSoft, Russia). The critical level of significance for testing statistical hypotheses used in this study was $\leq 0.05$. To determine the relationship between multiple variables, we used Friedman ANOVA and Kendall's concordance and Wilcoxon-matched pairs test.

\section{RESULTS}

Ultrasound examination of the right IJV was carried out in twenty adult patients. The demographic data of the patients is summarized in Table 1.

The data show that when gradual change the tilt angle from $0^{\circ}$ to $-20^{\circ}$ the IJV diameter is increased and IJV collapsibility index is decreased [Table 2]. Statistical analysis of changes of confident interval (CI) using Friedman ANOVA and Kendall's concordance provided the following results: $\chi^{2}(n=20)=70.86, P<0.00000$. Kendall coefficient of concordance $=0.88578$, with average rank $r=0.87977$. During paired testing of CI values at $0^{\circ}$ with $\mathrm{CI}$ values at $5^{\circ}, 10^{\circ}, 15^{\circ}$, and $20^{\circ}$ using a Wilcoxon-matched pairs test, we obtained the following results: $P=0.000196$, $P=0.000089, P=0.000089, P=0.000089$, respectively.
Thus, the hypothesis that differences exist between the study groups is accepted.

At $15^{\circ}$ tilt angle, the values of the vein diameter during inhalation and exhalation were not significantly different, indicating the absence of respiratory-based IJV excursion. Further increase in the tilt angle up to $20^{\circ}$ did not change these data significantly. Similarly, significant changes have not been observed when comparing the $\mathrm{CI}$ values at $15^{\circ}$ and $20^{\circ}$ tilt angles (CI: $8.2 \pm 6.8$ and $8.0 \pm 6.8$ correspondingly, $P>0.05$ ). Thus, a conclusion about the effectiveness of head-down tilt position can be justified when the value of collapsibility index is $<9 \%$.

In different patients, collapsibility index of $<9 \%$ was recorded at different head-down tilt angles [Table 3].

Based on these data, we found that collapsibility index of $<9 \%$ indicating the absence of respiratory-based IJV excursion was recorded in $25 \%$ of patients in the horizontal supine position. In this case, placing the patients in the head-down tilt position for IJV catheterization may not be indicated. In $65 \%$ of the patients, respiratory-based excursion was not observed at $10^{\circ}$ head-down tilt position. Only $35 \%$ of the patients required $15^{\circ}$ head-down tilt position.

\begin{tabular}{|c|c|}
\hline Study sample characteristics & Number (\%) \\
\hline \multicolumn{2}{|l|}{ General demographics } \\
\hline Number of patients & 20 \\
\hline Gender, \%female & $8 / 12(60 \%)$ \\
\hline Mean age \pm SD (years) & $51.4 \pm 11.2$ \\
\hline [median, range] & {$[53.5,30-65]$} \\
\hline
\end{tabular}

SD: Standart deviation

Table 2: Changes in internal jugular vein diameter versus head-down tilt positions angle

\begin{tabular}{lccccc}
\hline \multirow{2}{*}{$\begin{array}{l}\text { IJV diameter } \\
\text { and collapsibility } \\
\text { index }\end{array}$} & \multicolumn{5}{c}{ Head-down tilt positions angle, degrees } \\
\cline { 2 - 6 } & $\mathbf{0}^{\circ}$ & $\mathbf{5}^{\circ}$ & $\mathbf{1 0}^{\circ}$ & $\mathbf{1 5}^{\circ}$ & $\mathbf{2 0}^{\circ}$ \\
\hline $\begin{array}{l}\text { Dmin, mm } \\
\text { Mean } \pm \mathrm{SD}\end{array}$ & $6.1 \pm 4.0$ & $7.3 \pm 3.5$ & $9.0 \pm 2.9$ & $9.3 \pm 2.9$ & $9.3 \pm 2.8$ \\
Median & 5.4 & 7.5 & 8.9 & 9.3 & 9.3 \\
Dmax, mm & $8.6 \pm 3.3$ & $9.3 \pm 3.0$ & $9.9 \pm 2.8$ & $10.0 \pm 2.8$ & $10.1 \pm 2.7$ \\
Mean \pm SD & & & & & \\
Median & 8.6 & 9.7 & 9.9 & 10 & 10 \\
CI,\% Mean \pm SD & $36.0 \pm 29.6$ & $24.8 \pm 17.2$ & $9.6 \pm 8.9$ & $8.2 \pm 6.8$ & $8.0 \pm 6.8$ \\
Median & 29 & 19.5 & 8 & 7.3 & 7.2 \\
\hline
\end{tabular}

IJV: Internal jugular vein, SD: Standart deviation

Table 3: The occurrence of $\mathrm{Cl}<9 \%$ at different headdown tilt angles

\begin{tabular}{lccccc}
\hline \multirow{2}{*}{$\begin{array}{l}\text { Number of } \\
\text { patients (\%) }\end{array}$} & \multicolumn{5}{c}{ Head-down tilt positions angle, degrees } \\
\cline { 2 - 6 } & $\mathbf{0}^{\circ}$ & $\mathbf{5}^{\circ}$ & $\mathbf{1 0 ^ { \circ }}$ & $\mathbf{1 5}^{\circ}$ & $\mathbf{2 0}^{\circ}$ \\
\hline & $5(25 \%)$ & $5(25 \%)$ & $13(65 \%)$ & $12(65 \%)$ & $20(100 \%)$ \\
\hline
\end{tabular}




\section{Discussion}

Current practice guidelines for central venous access recommend that a patient should be placed in the head-down tilt position. ${ }^{[2]}$ It is believed that the right IJV increases in diameter and CSA to a greater extent when adult patients are placed in the head-down tilt position. Based on the common recommendations, $15^{\circ}-25^{\circ}$ head-down tilt positions are considered acceptable. ${ }^{[7,8]}$ However, there are no clear recommendations for selecting individual head-down tilt angle, which would provide safe and efficient maneuver for the particular patient. Some limitations and risks of complications are reported to be associated with the head-down tilt position. In this regard, we propose a method to determine if the placement in the head-down tilt position is indicated for the patient for IJV catheterization and to find the correct head-down tilt angle. The method is based on the fact that IJV diameter changes depending on the respiration phase. ${ }^{[4]}$ During the exhalation of a person, the diameter of veins increases, and during the inhalation, it decreases up to the total disappearing of their lumen that is strongly marked in patients with shock. ${ }^{[8]}$ It is known that during inhalation the vein diameter may decrease up to the complete disappearance of the vein lumen due to its collapse. IJV collapse may result in its perforation and damage to adjacent anatomical structures. Besides, vein collapse indicates a drop in blood pressure inside it. Moreover, the pressure in the vein at the moment of collapse may be lower than atmospheric pressure, which increases the risk of air embolism in the patient. Therefore, the absence of changes in the vein diameter during breathing and prevention of its collapse during inhalation can be considered as criteria for the head-down tilt position effectiveness and may reduce complications. The absence of changes in IJV diameter during breathing can be stated if collapsibility index is $<9 \%$. The results of our studies have shown that respiratory-based IJV excursion was recorded in $75 \%$ of patients in the horizontal supine position. Placing the patients in the head-down tilt position resulted in the disappearance of the respiratory-based vein excursion [Figure 1].
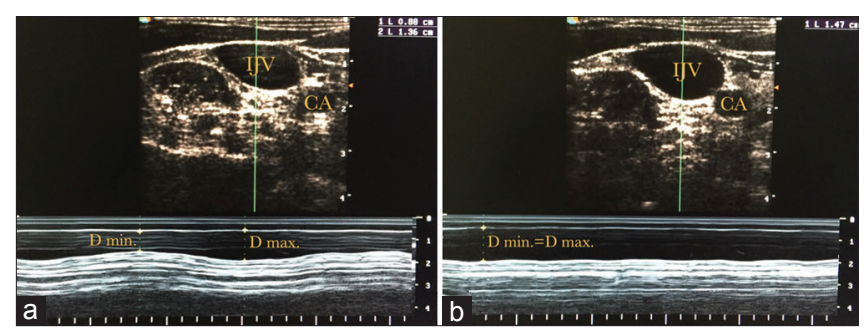

Figure 1: Measuring the right internal jugular vein diameter in adult patient using M-mode ultrasonography at $0^{\circ}$ (a) and $10^{\circ}$ (b) head-down tilt position.
The absence of the vein excursion on the ultrasound scanner screen was recorded in patients at different head-down tilt angles, in the range of $0^{\circ}-20^{\circ}$. After the disappearance of respiratory-based vein excursion, further lowering of head of the bed did not lead to a significant increase in the vein diameter, making it impractical. Thus, the absence of respiratory-based vein excursion in $25 \%$ of patients in the horizontal supine position can be a reason for not using the head-down tilt position during vein catheterization.

Our findings suggest the possibility of using ultrasonography to record the respiratory-based IJV excursion and the moment of its disappearance associated with the change in head-down tilt angle. In clinical settings, the disappearance of respiratory-based vein excursion on the ultrasound scanner screen can be considered as criteria of the head-down tilt position effectiveness. The proposed method is highly reproducible in clinical settings. Although further prospective studies are needed to improve the accuracy of this method, this research provides an important ground for carrying out similar clinical studies in future.

\section{Financial support and sponsorship}

Nil.

\section{Conflicts of interest}

There are no conflicts of interest.

\section{References}

1. Armstrong PJ, Sutherland R, Scott DH. The effect of position and different manoeuvres on internal jugular vein diameter size. Acta Anaesthesiol Scand 1994;38:229-31.

2. American Society of Anesthesiologists Task Force on Central Venous Access, Rupp SM, Apfelbaum JL, Blitt C, Caplan RA, Connis RT, et al. Practice guidelines for central venous access: A report by the American Society of Anesthesiologists Task force on central venous access. Anesthesiology 2012;116:539-73.

3. Onal O, Apiliogullari S, Nayman A, Saltali A, Yilmaz H, Celik JB. The effectiveness of trendelenburg positioning on the cross-sectional area of the right internal jugular vein in obese patients. Pak J Med Sci 2015;31:770-4.

4. Kasatkin AA, Urakov AL, Nigmatullina AR. Inventors. Method for Internal Jugular Vein Catheterization. Russian Federation Patent RU 2573337; 20 January, 2016.

5. Kasatkin AA, Urakov AL, Nigmatullina AR. Venous catheterization with ultrasound navigation. AIP Conf Proc 2015;1688:060010.

6. Gok F, Sarkilar G, Kilicaslan A, Yosunkaya A, Uzun ST. Comparison of the effect of the Trendelenburg and passive leg raising positions on internal jugular vein size in critically ill patients. Int J Clin Exp Med 2015;8:19037-43.

7. Suarez T, Baerwald JP, Kraus C. Central venous access: The effects of approach, position, and head rotation on internal jugular vein cross-sectional area. Anesth Analg 2002;95:1519-24.

8. Bellazzini MA, Rankin PM, Gangnon RE, Bjoernsen LP. Ultrasound validation of maneuvers to increase internal jugular vein cross-sectional area and decrease compressibility. Am J Emerg Med 2009;27:454-9. 